\title{
O ATUAL PAPEL DAS PROPRIEDADES NUTRICIONAIS DOS ALIMENTOS NA SAÚDE DAS POPULAÇÕES
}

\author{
THE CURRENT ROLE OF NUTRITIONAL PROPERTIES IN FOODS IN \\ THE HEALTH OF POPULATIONS
}

\author{
Aleson Pereira de Sousa ${ }^{1}$ \\ Laísa Vilar Cordeiro ${ }^{2}$ \\ Pedro Thiago Ramalho de Figueiredo ${ }^{3}$ \\ Giulian César da Silva Sá ${ }^{4}$ \\ Thamara Rodrigues de Melo ${ }^{5}$
}

RESUMO: Objetivo: A pesquisa pretende realizar uma revisão de literatura sobre o papel das propriedades nutricionais dos alimentos e o atual impacto na saúde das populações. Métodos: Foi realizada uma pesquisa do tema em revistas eletrônicas de cunho científico sendo elas Scientific Eletronic Library Online (SciELO), National Library of Medicine (PubMed) e Medline, além de sites institucionais e livros para a obtenção de conteúdo científico sobre as propriedades nutricionais dos alimentos e a saúde das populações. Resultados: Há grande desafios impostos para os profissionais de saúde aliarem o campo da nutrição no contexto da promoção da saúde. No entanto, tais desafios apontam para a possibilidade de compatibilizar diferentes olhares e perspectivas sobre uma atuação interdisciplinar. Trazer e incentivar o reconhecimento da alimentação saudável como medida de promoção da saúde não pode ser dissociada da adoção de hábitos de vida saudáveis, especialmente a prática de atividade física. Considerações Finais: As políticas de saúde e a população passam por constantes processos de mudança, onde primeiro havia um processo curativo de saúde visando apenas tratamento de patologias, hoje já torna-se antiquado trazer o processo de saúde apenas em caráter preventivo, atualmente busca-se exercer a promoção da saúde na prática profissional. Utilizar o

\footnotetext{
1 Doutorando do programa de Desenvolvimento e Inovação Tecnológica de Medicamentos pela Universidade Federal da Paraíba - UFPB. E-mail: aleson.pereira.sousa@gmail.com.

2 Doutoranda do programa de Produtos Naturais e Sintéticos Bioativos pela Universidade Federal da Paraíba - UFPB.

${ }^{3}$ Doutorando do programa de Produtos Naturais e Sintéticos Bioativos pela Universidade Federal da Paraíba - UFPB.

4 Doutorando programa de Bioquímica pela Universidade Federal do Rio Grande do Norte - UFRN.

${ }^{5}$ Doutoranda do programa de Produtos Naturais e Sintéticos Bioativos pela Universidade Federal da Paraíba - UFPB.
} 
prévio conhecimento da população para despertar a melhoria dos hábitos de vida garantindo melhores condições de saúde.

Palavras chave: Propriedades Nutricionais. Alimentos. Saúde das Populações.

ABSTRACT: Objective: The research intends to carry out a literature review on the role of the nutritional properties of foods and the current impact on the health of populations. Methods: Research on the topic was carried out in scientific journals of a scientific nature, Scientific Electronic Library Online (SciELO), National Library of Medicine (PubMed) and Medline, in addition to institutional websites and books for obtaining scientific content on nutritional properties. of food and the health of populations. Result: There are major challenges for health professionals to combine the field of nutrition in the context of health promotion. However, such challenges point to the possibility of making different perspectives and perspectives compatible with an interdisciplinary approach. Bringing and encouraging the recognition of healthy eating as a health promotion measure cannot be dissociated from the adoption of healthy lifestyle habits, especially the practice of physical activity. Final Considerations: Health policies and the population undergo constant processes of change, where first there was a curative health process aimed only at treating pathologies, today it is old-fashioned to bring the health process only in a preventive character, currently promoting health in professional practice. Use the population's prior knowledge to awaken the improvement of life habits ensuring better health conditions.

Keywords: Nutritional Properties. Food. Population Health. 


\section{INTRODUÇÃO}

O grande aumento populacional e maiores índices da expectativa de vida influenciam diretamente na necessidade de ampliação dos recursos em saúde. As possibilidades de tratamentos de doenças, especialidades na saúde, novas tecnologias empregadas para diagnóstico rápidos e eficazes, assim obtemos um cenário positivo e otimista para saúde acompanhar a velocidade nas mudanças populacionais (MIRANDA; MENDES; SILVA, 2016; MELO et al., 2019; CORDEIRO et al., 2019).

Portanto, os principais desafios na formação de novos profissionais de saúde encontram-se na: conceitos de saberes teórico/prático com atuação multidisciplinar, atualização e capacitação continuada, exercício com métodos de resolução de problemas de forma ativa. $O$ atual profissional de saúde deve estar atento a idealização de projetos e ações integradas em saúde, colaborando para construção e ratificação do conhecimento popular/científico, visando uma maior funcionalidade dos serviços de saúde prestados à população (PAUL, 2013; MELO et al., 2019).

A educação nutricional no Brasil também passa por constantes desafios, as práticas desenvolvidas pelos nutricionistas revelam a fragilidade de integração com disciplinas de cunho social, isto porque a contextualização dos problemas alimentares são diversificados e possuem abordagens diferenciadas nem sempre ao alcance da população tratada (MATTOS, 2004).

O método tradicional de educação nutricional se concentra nos efeitos biológicos dos nutrientes no organismo, orientação alimentar, preservação das propriedades nutritivas dos alimentos envolvendo as técnicas de preparo e armazenamento. Tais ações podem ser consideradas benéficas já que, em algumas circunstâncias, verifica-se que os indivíduos apresentam desconhecimento sobre a questão alimentar (FERREIRA; MAGALHÃES, 2007).

Entretanto, o Brasil é um país extenso com diversidade cultural e de grandes contrastes na desigualdades social, compromentendo o acesso da população ao 
direito universal à alimentação. A desigualdade no acesso ao alimento é uma realidade vivenciada por parcelas significativas dos brasileiros (STRINGHETA et al., 2007).

Neste sentido, torna-se relevante avaliar o atual papel das propriedades nutricionais dos alimentos na saúde das populações, onde o presente estudo objetivou realizar uma revisão de literatura traçando os elementos sobre a diversidade de conhecimento e atuação dos profissionais de saúde sobre a ingesta de alimentos trazendo os benefíficios e malefícios para população brasileira.

\section{MÉTODOS}

O presente trabalho trata-se de uma revisão bibliográfica descritiva de caráter exploratório dedutivo, realizada por meio da seleção de estudos na área e interpretação das informações, utilizando como instrumentos para coleta de dados as referências eletrônicas SciELO (Scientific Eletronic Library Online), BVS Brasil (Biblioteca Virtual em Saúde) e PubMED, afim de selecionar publicações nacionais e internacionais, dentre os anos de 1980 a 2019, para isso serão utilizados os descritores: propriedades nutricionais, alimentos, saúde das populações.

Após realização da busca e leitura dos artigos, os mesmos foram organizados de acordo com os seguintes critérios de inclusão: terem sido publicados entre o período de 1980 e 2019; serem estudos observacionais ou aplicados e de campo; serem estudos nacionais e internacionais terem em seus objetivos a problemática ou tema condizente ao proposto no presente estudo, foram excluídos artigos que não apresentaram a versão completa para leitura, os trabalhos publicados fora do período proposto. 


\section{RESULTADOS E DISCUSSÃO}

\section{Desenvolvimento Populacional}

O panorama atual de crescimento da população em nível mundial tem vivido um processo de transição demográfica muito importante, o chamado envelhecimento populacional, que se deu mediante ao aumento gradual da expectativa de vida que é resultado principalmente da diminuição das taxas de natalidade e mortalidade (SERBIM; FIGUEIREDO, 2011).

O desenvolvimento de novas tecnologias tem gerado melhorias em vários setores: econômico, social e na saúde. O desenvolvimento atingi todas as camadas trazendo mais benefícios e também maiores desafios, uma vez que o cenário muda sua problemática também assume novo contexto (CORDEIRO et al., 2019).

Esse envelhecimento populacional ocasionou uma mudança nos padrões de morbimortalidade, a chamada transição epidemiológica, onde as doenças infecciosas deram lugar às doenças crônicas não transmissíveis (DCNT) como causas de morbimortalidade (TELESI-JUNIOR, 2016; SUZMAN; HAAGA, 2013). Onde a população muda seu conceito de saúde, passando do processo curativo para o modelo de prevenção, buscando maior qualidade de vida.

O crescimento acelerado do número de senis na população mundial tem sido considerado como um fenômeno desafiador, pois instiga mudanças nas politicas de saúde à fim de garantir o cuidado com a saúde da pessoa idosa principalmente quando se necessita de readptações alimentares para promover melhor qualidade de vida na população (MIRANDA; MENDES; SILVA, 2016; CORDEIRO et al., 2019). 


\section{A promoção da saúde e política nacional brasileira}

O acesso à saúde no Brasil teve grande modificações e tornou-se um direto social através do Sistema Único de Saúde (SUS), concretizado a partir da Constituição Federal de 1988 (CF/88) (VIACAVA et al.; 2018). O artigo 196 da Constituição Federal de 1988 destaca que: "saúde é direito de todos e dever do estado" (BRASIL; 2003).

O principal objetivo é realizar práticas que visem à redução do risco de doenças e agravos com ações e serviços de promoção, proteção e recuperação da saúde, obedecendo ao nível de regionalização e hierarquização para efetuar o serviço através da organização apresentada pelas diretrizes de descentralização, atendimento integral e participação social, decretado no artigo 198 da CF/88 (BRASIL; 2003; COSTA et al., 2019).

Em todo o mundo há interesse em conquistar melhores hábitos de saúde e trazer a qualidade vida, a nutrição é um foco importante para garantir a redução de gastos com processos de doença na população. Por meio do incentivo a prevenção de doenças crônicas, da melhoria da qualidade e estímulo para maior expectativa de vida ativa (STRINGHETA et al., 2007).

As políticas de saúde brasileiras referentes a alimentação e nutrição seguem a tendência das recomendações na estratégia global sobre dieta, atividade física e saúde, segundo a Organização Mundial da Saúde (WHO, 2004). A estratégia recomenda aos países-membros incentivo de políticas que estimulem a alimentação saudável, prática de atividade física, modificando o quadro de ocorrências sobre doenças crônicas não-transmissíveis causadas pela má alimentação e por estilo de vida sedentário (STRINGHETA et al., 2007; COSTA et al., 2019).

O Ministério da Saúde em 10 de junho de 1999 aprovou a criação do Plano Nacional de Alimentação e Nutrição (PNAN), sua aprovação pelo Conselho Nacional de Saúde do Brasil teve a Portaria $n^{\circ} 710$ (Brasil, 1999). O propósito de garantir a qualidade dos alimentos colocados para consumo no país, a promoção de práticas alimentares saudáveis e a prevenção e o controle dos distúrbios nutricionais, bem 
como o estímulo às ações intersetoriais que propiciem o acesso universal aos alimentos (STRINGHETA et al., 2007).

Dessa forma, o surgimento de novas políticas visa incentivar e direcionar os profissionais de saúde aos novos desafios que as mudanças sociodemográficas apresentam com crescimento de populações, A Figura 1 ilustra as interfaces da Política Nacional de Alimentação e Nutrição e Política Nacional de Promoção da Saúde com a Política Nacional de Saúde.

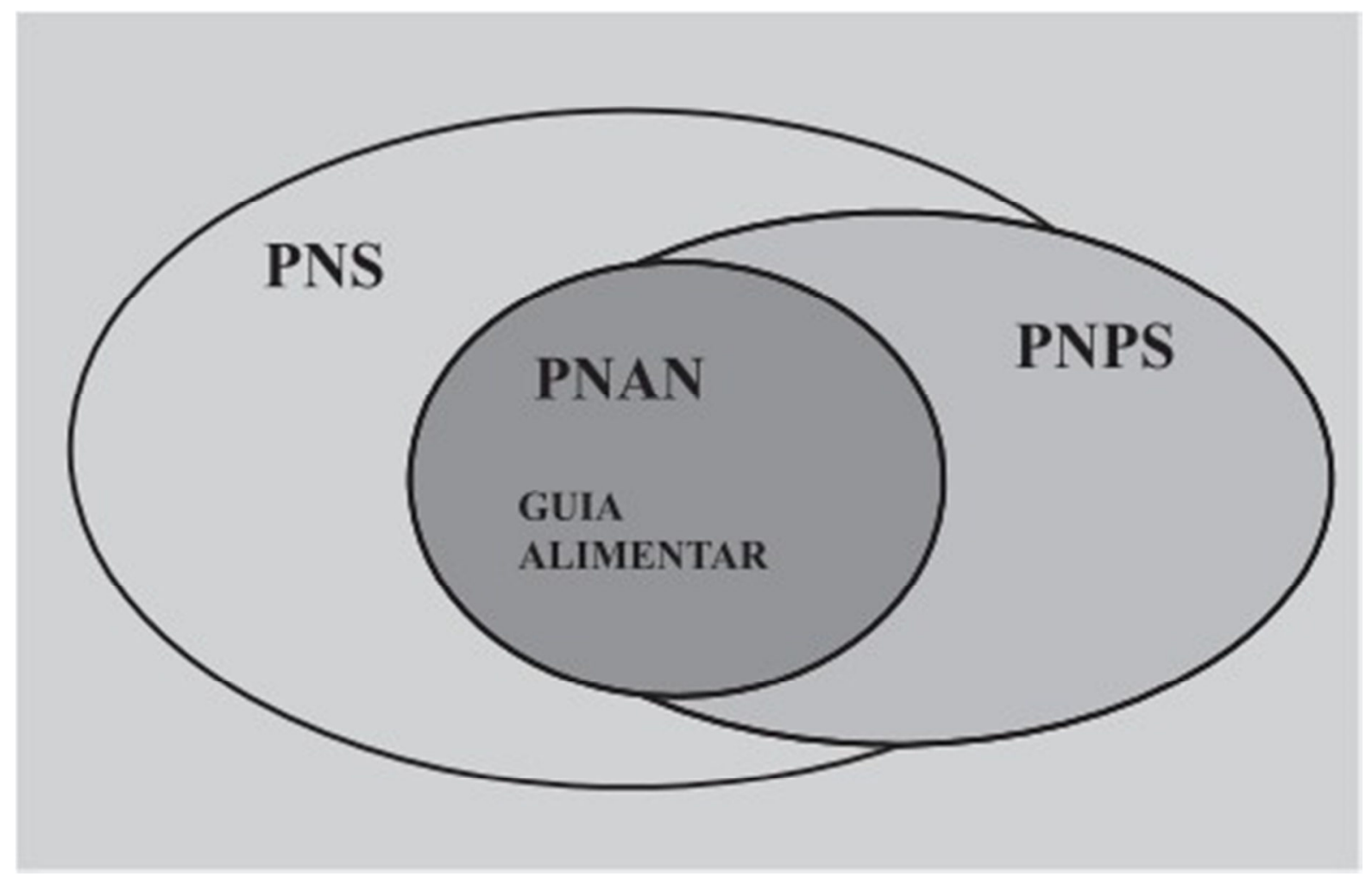

Figura 1. Interfaces das Políticas Nacionais Brasileiras de Saúde (PNS), de Alimentação e Nutrição (PNAN), Guia Alimentar para a População Brasileira (GAPB), e de Promoção da Saúde (PNPS) - Fonte: adaptada de Oliveira (2006).

O aumento da demanda no ramo da saúde ocasionado, principalmente, por doenças crônicas, o alto custo dos serviços de saúde, descontentamento com os ofícios de saúde vigentes, tem elevado o interesse por uma assistência holística e preventiva aos agravos e as terapêuticas que ofertem qualidade de vida aos indivíduos, promovendo a procura de outras maneiras de efetivar o cuidado (SANTANA, 2016; MELO et al., 2019). 
A inclusão do campo da alimentação e nutrição no debate da promoção da saúde aponta para avanços importantes na conquista de atenção e qualidade de vida das populações, trazendo novos horizontes para a atuação dos profissionais de saúde, principalmente os nutricionistas sociais. Por um lado, há possibilidade de repensar as práticas de atuação profissional na saúde no campo da saúde pública, de outro lado temos importantes percalços a serem constantemente superados (FERREIRA; MAGALHÃES, 2007).

\section{Propriedades nutricionais dos alimentos}

Substâncias químicas presentes em alimentos, os nutrientes, são necessárias à vida. Essas possuem funções cruciais às células que constituem os organismos, tais como: crescimento, manutenção e reparo celular. Os nutrientes são divididos em dois grupos: os macronutrientes (proteínas, lípidos e carboidratos) e os micronutrientes (vitaminas, minerais, aminoácidos essenciais e ácidos graxos). A ingestão e absorção de todos os nutrientes é essencial à promoção e manutenção da saúde ao longo do período de vida, devendo ser equilibrada e realizada em função das necessidades do organismo de modo a reduzir a prevalência de diversas doenças (STIPANUK; CAUDILL, 2013; SOUSA et al., 2017).

Baseado nessa premissa, estudos relacionados ao consumo de alimentos com propriedades nutricionais que contribuam para a saúde das populações, ingestão de nutrientes que apresentam alto teor de proteínas, vitaminas e minerais; tem sido considerado de grande importância para a comunidade (VIDAL et al., 2012). Todavia, algumas moléculas presentes nestes alimentos, podem apresentar substâncias consideradas antinutritivas e/ou tóxicas, como cianeto, polifenóis, nitrato, ácido oxálico, saponinas, inibidores de proteases e as lectinas (SILVA et al., 2010; SOUSA, 2017).

Dentre tais fatores antinutricionais, os principais de natureza proteica são os inibidores de proteases e as lectinas, os quais afetam o metabolismo basal e/ou 
crescimento de diferentes espécies animais (PUSZTAl et al., 1997; ARMOUR et al., 1998; SILVA; SILVA, 2000).

Os efeitos nocivos dos inibidores de proteases presentes em alimentos são complexos. Vários estudos têm atribuído efeitos deletérios, principalmente alterações metabólicas do pâncreas (aumento da secreção enzimática, hipertrofia e hiperplasia) e redução da taxa de crescimento, à presença de inibidores de proteases em fontes alimentícias provenientes de leguminosas e hortaliças (ALETOR; ADEOGUN, 1995; SILVA et al., 2010).

Uma dieta equilibrada deve conter proteínas, sobretudo, as de origem animal, que possuem maior valor biológico em relação as advindas de fontes vegetais. Porém, as populações de baixo poder aquisitivo têm acesso limitado a alimentos ricos em proteínas de origem animal. A identificação de espécies vegetais ricas em proteínas e incentivos de cultivo e consumo destas espécies podem vir a contribuir para diminuir as deficiências nutricionais das populações fornecendo alternativas nutricionais variadas, especialmente para aqueles com hábitos e dietas alimentares diferenciados (ASZTALOS et al., 2006).

As proteínas são macromoléculas constituídas pela união de aminoácidos exercendo funções biológicas diversas nas células e organismos. Tais macromoléculas podem se diversificar através da sequência aminoacídica e conformação tridimensional da cadeia peptídica, resultante do processo de formação da proteína. Sendo este o principal objeto de estudo de químicos e bioquímicos ao longo dos anos. As moléculas proteicas são capazes de ligar substratos, transportar informações e moléculas para as células, ativar ou inibir substratos exercendo funções cruciais na atividade enzimática dos organismos (SHARON; LIS, 2001).

As proteínas extraídas principalmente das folhas das plantas são denominadas leaf proteins (proteínas foliares) e são encapsuladas e utilizadas por adeptos a dietas ricas em alimentos naturais, especialmente no caso de consumo das plantas, herbáceas, podendo ser consumido/extraído das plantas inteiras, trazendo vários benefícios para saúde da população (FERNANDO, 1980).

Além do interesse do ponto de vista nutricional, tais moléculas biológicas têm sido bastante exploradas quanto ao seu potencial em aplicações biotecnológicas 
envolvendo as áreas de agropecuária, nutrição, saúde e produtos químicos industriais (VIDAL et al., 2012).

A busca por aplicações e descobertas destes novos produtos naturais envolve a investigação biológica da estrutura de carboidratos presentes em diversas células (SILVA; SILVA, 2000). Para esse tipo de estudos, utiliza-se matrizes cromatograficas comerciais de afinidade na caracterização e purificação destes polissacarídeos e/ou glicoconjugados (LIMA et al., 1997).

Neste contexto, as lectinas, moléculas proteicas bioativas advindas de produtos naturais, têm sido amplamente empregadas na caracterização de grupos sanguíneos, principalmente por bancos de sangue (SHARON; LIS, 1998). Ainda verifica-se que as lectinas possam estimular da mitogênese de linfócitos, abrindo novas perspectivas no campo da imunologia (SHARON; LIS, 2004). Assim como, atuar sobre as células cancerígenas, sendo utilizadas nos estudos de oncogênese (SHARON; LIS, 2004); também são utilizadas como agentes defensivos na agricultura, em função de sua ação fungicida, bactericida e inseticida (GAIDAMASHVILI; VAN STADEN, 2002; PEUMANS et al., 2000).

Produtos naturais contendo fatores antinutricionais como lectinas podem reconhecer resíduos de carboidratos presentes nas células intestinais, exercendo sua função de ligação. Devido a ligação destas moléculas aos receptores glicosilados das células intestinais, tais proteínas interferem na digestão, absorção e utilização de nutrientes, devido à paralisação do transporte dos nutrientes e à absorção de substâncias nocivas. No entanto, o exato mecanismo de ação de muitas dessas moléculas ainda não foram elucidados (CZAPLA, 1997; NEVEL et al., 1998; TINJUANGUJUN, 2002).

\section{CONSIDERAÇÕES FINAIS}

Há grande desafios impostos para os profissionais de saúde aliarem o campo da nutrição no contexto da promoção da saúde. No entanto, tais desafios apontam para a possibilidade de compatibilizar diferentes olhares e perspectivas sobre uma 
atuação interdisciplinar. Trazer e incentivar o reconhecimento da alimentação saudável como medida de promoção da saúde não pode ser dissociada da adoção de hábitos de vida saudáveis, especialmente a prática de atividade física.

As políticas de saúde e a população passam por constantes processos de mudança, onde primeiro havia um processo curativo de saúde visando apenas tratamento de patologias, hoje já torna-se antiquado trazer o processo de saúde apenas em caráter preventivo, atualmente busca-se exercer a promoção da saúde na prática profissional. Utilizar o prévio conhecimento da população para despertar a melhoria dos hábitos de vida garantindo melhores condições de saúde.

\section{REFERÊNCIAS BIBLIOGRÁFICAS}

ALETOR, V. A.; ADEOGUN, O. A. Nutrient and anti-nutrient components of some tropical leafy vegetables. Food chemistry, v. 53, n. 4, p. 375-379, 1995.

ARMOUR, J. C., PERERA, R. C., BUCHAN, W. C., GRANT, G. Protease inhibitors and lectins in soya beans and effects of aqueous heat-treatment. Journal of the Science of Food and Agriculture, v. 78, n. 2, p. 225-231, 1998.

ASZTALOS, B. F., SCHAEFER, E. J., HORVATH, K. V., COX, C. E., SKINNER, S., GERRIOR, J., WANKE, C. Protease inhibitor-based HAART, HDL, and CHD-risk in HIV- infected patients. Atherosclerosis, v. 184, n. 1, p. 72-77, 2006.

BRASIL; Conselho Nacional de Secretários de Saúde, Constituição Federal de 1988. In: Legislação do SUS. Brasilia: CONASS, 2003. p. 20-21.

CORDEIRO, A. S. R., PAUlA, C. L. M., COSTA, M. M., DANTAS, T. M., SOUSA, A. P. Internações e mortalidade por quedas em idosos no Brasil: revisão de literatura. Revista COOPEX/FIP. 10ª Edição - Vol. 10 - Ano: 2019.

COSTA, M. M., CORDEIRO, A. S. R., MEDEIROS, W. S. A., SOUSA, A. P. A importância da integralidade e humanização nos atendimentos públicos de saúde. Revista COOPEX/FIP. 10a Edição - Vol. 10 - Ano: 2019.

CZAPLA, T. H. Plant lectins as insect control proteins in transgenic plants. In: AdVances in insect control: The role of transgenic plants. CRC Press, 1997.

FERNANDO, R. Alimentos tradicionales y no tradicionales. Roma: FAO, 168 p., 1980.

FERREIRA, V. A., MAGALHÃES, R. Nutrição e promoção da saúde: perspectivas atuais. Cadernos de Saúde Pública, v. 23, p. 1674-1681, 2007.

GAIDAMASHVILI, M.; VAN STADEN, J.; ELOFF, J. N. Lectin-like proteins from South African plants used in traditional medicine. South African journal of botany, v. 68, n. 1, p. 36-40, 2002. 
LIMA, V. L., CORREIA, M. T., CECHINEL, Y. M., SAMPAIO, C. A., OWEN, J. S., COÊLHO, L. C. Immobilized Cratylia mollis lectin as a potential matrix to isolate plasma glycoproteins, including lecithin-cholesterol acyltransferase. Carbohydrate Polymers, v. 33, n. 1, p. 27-32, 1997.

MATTOS, R. A. D. A integralidade na prática (ou sobre a prática da integralidade). Cadernos de Saúde Pública, v. 20, p. 1411-1416, 2004.

MELO, V. P., BARBOZA, J. P., DANTAS, T. M., DANTAS, V. M. C., ARAÚJO, F. N., SOUSA, A. $P$. Práticas integrativas e complementares: prevenção e tratamento na atenção básica de saúde. Revista COOPEX/FIP. 10ª Edição - Vol. 10 - Ano: 2019.

MIRANDA, G. M. D., MENDES, A. C. G., SILVA, A. L. A. O envelhecimento populacional brasileiro: desafios e consequências sociais atuais e futuras. Revista Brasileira de Geriatria e Gerontologia, Rio de Janeiro, v. 19, n. 3, p. 507-519, 2016.

Ministério da Saúde. Portaria $n^{\circ} 710$, de 10 de junho de 1999. Aprova a Política Nacional de Alimentação e Nutrição. Diário Oficial da República Federativa do Brasil. Brasília, 11 jun. 1999. 1999.

NEVEL, C. V., DE RYCKE, H., BEECKMANS, S., DE WILDE, R., VAN DRIESSCHE, E. Inhibitory action of spray dried blood plasma and whole egg powder on lectins in extracts of several legume seeds: a qualitative approach. Journal of the Science of Food and Agriculture, v. 77, n. 3, p. 319-326, 1998.

OLIVEIRA, T.P. A aplicação da Política Nacional de Alimentação e Nutrição. In: I Congresso Internacional Sobre Alimentos Funcionais - Ciência, Inovação e Regulamentação. São Paulo. abril. 2006. 2020.

PAUL, P. Saúde e transdisciplinaridade. São Paulo: Edusp, 2013.

PEUMANS, W. J., ZHANG, W., BARRE, A., ASTOUL, C. H., BALINT-KURTI, P. J., ROVIRA, P., VAN DAMME, E. J. Fruit-specific lectins from banana and plantain. Planta, v. 211, n. 4, p. 546$554,2000$.

PUSZTAI, A., GRANT, G., BARDOCZ, S., GELENCSER, E., HAJOS, G. Y. Novel dietary strategy for overcoming the antinutritional effects of soyabean whey of high agglutinin content. British Journal of Nutrition, v. 77, n. 06, p. 933-945, 1997.

SANTANA, C. P. V.; NASCIMENTO, A. Práticas Integrativas e complementares: cuidado integral dentro da atenção psicossocial através de práticas corporais. Bahia, 2016.

SERBIM, A. K.; FIGUEIREDO, A. E. P. L. Qualidade de vida de idosos em um grupo de convivência. Scientia Medica, Porto Alegre, v. 21, n. 4, p. 166-172, 2011.

SHARON, N., LIS, H. The structural basis for carbohydrate recognition by lectins. In: The Molecular Immunology of Complex Carbohydrates-2. Springer US, p. 1-16, 2001.

SHARON, N., LIS, H. History of lectins: from hemagglutinins to biological recognition molecules. Glycobiology, v. 14, n. 11, p. 53R-62R, 2004.

SHARON, N.; LIS, H. Lectins, New York, 126pp, Chapman and Hall. London, 1998.

SILVA, M. R., SILVA, M. A. A. P. D. Fatores antinutricionais: inibidores de proteases e lectinas. Revista de Nutrição, 2000.

SILVA, M. C., CORREAA, A. D., SANTOS, C. D. D., MARCOS, F. C. A., ABREU, C. M. P. 
D. Extração da lectina da folha de mandioca (Manihot esculenta Crantz) eo efeito de cátions divalentes na atividade hemaglutinante. Ciência e Tecnologia de Alimentos, v. 30, n. 1, p. 103-107, 2010.

SOUSA, A. P. Obtenção da fração proteica dioscorina de inhame (Dioscorea cayennensis): caracterização bioquímica e atividades biológicas. $65 \mathrm{f}$. Dissertação (Programa de Pós Graduação em Biologia Celular e Molecular) - Universidade Federal da Paraíba-PB, 2017.

SOUSA, A. P.; NASCIMENTO, E. S.; MADRUGA, M. S.; LIMA, E. O.; SILVA, D. F.; GADELHA, T. S.; GADELHA, C. A. A. Determinação do potencial antioxidante, antibacteriano e antifúngico da fração proteica dioscorina de inhame (Dioscorea cayennensis). Rev. Bras. PI. Med., São Paulo, v.19, n.3, p.463-471, 2017.

STIPANUK, M.; CAUDILL, M. Biochemical, Physiological, and Molecular Aspects of Human Nutrition. 3. ${ }^{a}$ ed. Elsevier Saunders: 968 p, 2013.

STRINGHETA, P. C., OliveIRA, T. T. D., GOMES, R. C., AMARAL, M. D. P. H. D., CARVALHO, A. F. D., VILELA, M. A. P. Políticas de saúde e alegações de propriedades funcionais e de saúde para alimentos no Brasil. Revista Brasileira de Ciências Farmacêuticas, v. 43, n. 2, p. 181-194, 2007.

SUZMAN, R.; HAAGA, J. G. Demografia Mundial do Envelhecimento. In: LONGO, D.L. (Org.). Medicina Interna de Harrison. v. 1. 15. ed. Porto Alegre: AMGH, 2013.

TELESI-JUNIOR, E. Práticas integrativas e complementares em saúde, uma nova eficácia para o SUS. Estud, v. 30, n. 86, p. 99-112, 2016.

TINKUANGJUN, P. Snowdrop lectin gene in transgenic plants: its potential for Asian agriculture. AgBiotechNet, wallingford, v.4, p. 1-5, 2002.

VIACAVA, F., OLIVEIRA, R. A. D., CARVALHO, C. C., LAGUARDIA, J., BELLIDO, J. G. SUS: oferta, acesso e utilização de serviços de saúde nos últimos 30 anos. Ciencia \& saude coletiva, v. 23, p. 1751-1762, 2018.

VIDAL, A. M., DIAS, D. O., MARTINS, E. S. M., OLIVEIRA, R. S., NASCIMENTO, R. M. S., DA SILVA CORREIA, M. D. G. A ingestão de alimentos funcionais e sua contribuição para a diminuição da incidência de doenças. Caderno de Graduação-Ciências Biológicas e da Saúde-UNIT, v. 1, n. 1, p. 43-52, 2012.

WORLD HEALTH ORGANIZATION .WHO. Global strategy on diet, physical activity and health: fiftyseventh World Health Assembly Wha 57.17. 22 May 2004. 2020. 